\title{
Population structure of Helicobacter pylori among ethnic groups in Malaysia: recent acquisition of the bacterium by the Malay population
}

\author{
Chin Yen Tay ${ }^{1}$, Hazel Mitchell1, Quanjiang Dong1, Khean-Lee Goh², \\ Ian W Dawes ${ }^{1}$ and Ruiting Lan*1
}

\begin{abstract}
Address: ${ }^{1}$ School of Biotechnology and Biomolecular Sciences, University of New South Wales, Sydney, NSW 2052, Australia and ${ }^{2}$ Department of Medicine, Faculty of Medicine, University of Malaysia, Kuala Lumpar, Malaysia

Email: Chin Yen Tay - alfred.tay@gmail.com; Hazel Mitchell - H.Mitchell@unsw.edu.au; Quanjiang Dong - jiangacer@126.com; KheanLeeGoh - gohkl@um.edu.my; IanW Dawes - i.dawes@unsw.edu.au; Ruiting Lan* - r.lan@unsw.edu.au

* Corresponding author
\end{abstract}

Published: 19 June 2009

BMC Microbiology 2009, 9:126 doi:10.1186/147|-2180-9-126

This article is available from: http://www.biomedcentral.com//47/-2/80/9//26

(C) 2009 Tay et al; licensee BioMed Central Ltd.

This is an Open Access article distributed under the terms of the Creative Commons Attribution License (http://creativecommons.org/licenses/by/2.0), which permits unrestricted use, distribution, and reproduction in any medium, provided the original work is properly cited.

\begin{abstract}
Background: Helicobacter pylori is a major gastric bacterial pathogen. This pathogen has been shown to follow the routes of human migration by their geographical origin and currently the global $H$. pylori population has been divided into six ancestral populations, three from Africa, two from Asia and one from Europe. Malaysia is made up of three major ethnic populations, Malay, Chinese and Indian, providing a good population for studying recent $\mathrm{H}$. pylori migration and admixture.
\end{abstract}

Results: Seventy eight $H$. pylori isolates, including 27 Chinese, 35 Indian and 16 Malay isolates from Malaysia were analysed by multilocus sequence typing (MLST) of seven housekeeping genes and compared with the global MLST data. STRUCTURE analysis assigned the isolates to previously identified $H$. pylori ancestral populations, hpEastAsia, hpAsia2 and hpEurope, and revealed a new subpopulation, hsplndia, within hpAsia2. Statistical analysis allowed us to identify population segregation sites that divide the $H$. pylori populations and the subpopulations. The majority of Malay isolates were found to be grouped together with Indian isolates.

Conclusion: The majority of the Malay and Indian $H$. pylori isolates share the same origin while the Malaysian Chinese $\mathrm{H}$. pylori is distinctive. The Malay population, known to have a low infection rate of $H$. pylori, was likely to be initially $H$. pylori free and gained the pathogen only recently from cross infection from other populations.

\section{Background}

Helicobacter pylori may have infected humans since their origin and currently is believed to infect more than half the population in the world $[1,2]$. Infection is usually acquired during childhood by intrafamilial transmission and in the majority of cases infection is lifelong unless eradication by antibiotic treatment is undertaken $[3,4]$.
The prevalence of $H$. pylori infection ranges from $25 \%$ in developed countries to more than $80 \%$ in the developing regions $[3,5,6] . H$. pylori is commonly transmitted from mother to child [3].

H. pylori is well known for being highly diverse and recombining frequently. DNA sequence analysis of house- 
keeping and virulence associated genes all have illustrated the unusually high degree of genetic variability in this species $[2,7-12]$. Comparison of isolates within a single host sampled over an average of 1.8 years has revealed that an average of 100 DNA imports occur between bacteria, corresponding to $3 \%$ of the genome or $50 \mathrm{~kb}[11]$ and by extrapolation from these data, it was predicted that within 41 years half the genome would have been replaced by imports [11]. In comparison, 10-100 million years were needed to replace $60 \%$ of the E. coli genome [13].

Studies suggest that recombination is rare between isolates from different continents and as such $H$. pylori behaves like a genetic marker of human descent and reflects the human population in which the host spent his/her childhood $[2,10,12]$. Multilocus sequence typing (MLST) of seven housekeeping genes from several hundred H. pylori strains isolated from different geographical, ethnic, and/or linguistic origins showed that $H$. pylori followed human migration out of Africa and identified six $H$. pylori populations which are designated as hpAfrica1, hpAfrica2, hpNEAfrica, hpEurope, hpEastAsia, and hpAsia2 $[2,12]$. Three of these populations are further divided into subpopulations: hpEastAsia is divided into three subpopulations, hspEAsia, hspAmerind and hspMaori. The hspMaori subpopulation has been isolated exclusively from Maoris and other Polynesians and the hspAmerind from Inuits and Amerinds in North and South America; hpAfrica1 is divided into hspSAfrica and hspWAfrica; hpEurope is divided into Ancestral European 1 (AE1) and Ancestral European 2 (AE2).

Countries with populations of multiple origins provide a good opportunity to further study the population structure of $H$. pylori. Malaysia is composed of three major ethnic populations: Malay (65\%), Chinese $(26 \%)$ and Indian (7.7\%) http://www.statistics.gov.my. The majority of Malaysian Chinese migrated from Southern China, the Malaysian Indians from Southern India and the Malays are in general considered natives of Malaysia [14]. The Malaysian Malay population is made up of a mixture of people extant in South East Asia as early as 3000 years ago [15]. However, in modern Malaysia they are now referred to as the Malays [16]. The aboriginal Orang Asli people in Malaysia do not share the same origin as the Malays [17].

H. pylori Infection is associated with an increased risk of developing peptic ulcer disease and gastric cancer $[18,19]$ as well as an increased risk of developing primary non-Hodgkin's lymphomas of the stomach (MALT lymphoma) [20]. Previous studies have shown that the Indian ethnic group has the highest rate of $H$. pylori infection (68.9-75\%), followed by the Chinese (45-60\%) and the Malay the lowest $(8-43 \%)[21,22]$. This difference of prevalence was also found in children [23]. Interestingly the three populations have different rates of gastric cancer. While the Malaysian Chinese population has a high incidence the Malaysian Indian population has a low incidence [24]. The phenomenon of high prevalence of $H$. pylori but low incidence of gastric cancer has been dubbed the "Indian Enigma" [24]. A better understanding of the population structure of $H$. pylori in these ethnic populations is clearly needed to order to elucidate the differences in infection rates and disease severity. We used MLST to analyse H. pylori isolates obtained from the three ethnic groups in Malaysia. We show the similarity between the Malay and the Indian H. pylori isolates and the diversity between the Malaysian Indian $H$. pylori population identified in this study and the Indian Ladakh $H$. pylori population identified by Linz et al. [2].

\section{Results \\ Nucleotide diversity of the housekeeping genes}

Fragments of seven housekeeping genes, atpA (566 bp), efp (350 bp), mutY (361 bp), ppa (338 bp), trpC (396 bp), ureI (525 bp), and $y p h C$ (450 bp), with a total length of 2,982 bp were sequenced from 78 Malaysian $H$. pylori isolates, including 27 Chinese, 16 Malay and 35 Indian isolates. MLST data of 423 isolates comprising of isolates from two studies by Achtman's group [2,12] available at the time of analysis were extracted from the H. pylori MLST database http://pubmlst.org/helicobacter/ and included in the analysis with data from this study. The level of nucleotide diversity between populations and between genes is shown in Table 1. The most diverse gene was trpC in all except the Malaysian Chinese population with the highest diversity at nearly $7.6 \%$ while the least diverse gene was atpA at $2.6 \%$. The three ethnic populations showed different levels of diversity with the Chinese population the lowest while the Indian and Malay populations were similar. All ethnic groups had lower level of variation than the global population as a whole.

\section{STRUCTURE analysis}

To determine the relationship of the Malaysian H. pylori isolates and the global isolates, we analysed our MLST data together with the global data using the Bayesian statistics tool, STRUCTURE [25], which was previously used to divide global $H$. pylori isolates into six ancestral populations, designated as hpAfrica1, hpAfrica2, hpNEAfrica, hpEurope, hpEastAsia and hpAsia2 [2,12]. The Malaysian $H$. pylori isolates were found to fall into four of the six known populations (Fig. 1A). Twenty three Indian and nine Malay isolates were grouped with hpAsia2; 26 Chinese, four Indian and two Malay isolates grouped with hpEastAsia; one Chinese, eight Indian and four Malay isolates grouped with hpEurope; and one Malay isolate grouped with hpAfrica1 (Fig. 1A). Phylogenetic analysis using the Neighbour joining algorithm as shown in Figure $1 \mathrm{~B}$ divided the isolates into three clusters, consistent with the STRUCTURE analysis. 
Table I: Sequence variation

\begin{tabular}{|c|c|c|c|c|c|c|c|c|c|}
\hline \multirow[t]{2}{*}{ Gene } & \multirow[t]{2}{*}{ Size (bp) } & \multicolumn{4}{|c|}{ Diversity (\%) } & \multicolumn{4}{|c|}{ Population segregation sites } \\
\hline & & Chinese (27) & Indian (35) & Malay (16) & Global (492) & $\begin{array}{l}\text { hspEAsia vs } \\
\text { hspMaori }\end{array}$ & $\begin{array}{l}\text { hspEAsia vs } \\
\text { hspAmerind }\end{array}$ & $\begin{array}{l}\text { hsplndia vs } \\
\text { hspEAsia }\end{array}$ & $\begin{array}{l}\text { hsplndia vs } \\
\text { hspLadakh }\end{array}$ \\
\hline atpA & 566 & 1.77 & 1.61 & 2.22 & 2.62 & 5 & 4 & 5 & 4 \\
\hline efp & 350 & 1.95 & 2.38 & 3.13 & 3.34 & 4 & 1 & 6 & 3 \\
\hline mutY & 361 & 3.62 & 4.85 & 4.49 & 6.5 & 8 & 7 & 9 & 7 \\
\hline ppa & 338 & 1.76 & 2.24 & 2.16 & 3.22 & 1 & 1 & 1 & 0 \\
\hline $\operatorname{trpC}$ & 396 & 3.35 & 6.78 & 6.91 & 7.6 & 9 & 16 & 16 & 16 \\
\hline urel & 525 & 2.08 & 2.39 & 2.66 & 3.21 & 9 & 9 & 8 & 5 \\
\hline yphC & 450 & 2.34 & 3.79 & 3.87 & 4.84 & 10 & 4 & 8 & 6 \\
\hline All seven & 2,980 & 2.37 & 3.35 & 3.55 & 4.33 & 39 & 32 & 48 & 27 \\
\hline
\end{tabular}

Since some populations can be further divided into subpopulations (Fig. 1A) [2,12], we used the same approach to further classify the Malaysian isolates into subpopulations. For the Malaysian isolates in the hpEastAsia population, the majority (26 Chinese, three Indian and one Malay) fell into hspEAsia except for two isolates (one Indian and one Malay) falling into the hspMaori subpopulation. hpAsia2 had previously no subpopulations. There were 77 isolates in hpAsia2 including 32 isolates from this study and 41 Ladakh isolates. Our STRUCTURE analysis divided these 77 isolates into two subpopulations (Fig. 2). All 41 Ladakh isolates were grouped as one subpopulation while the remaining 36 isolates including 32 Malaysian Indian and Malay isolates from this study, one Singapore isolate and three UK isolates (Bangladesh origin) grouped together as another (Fig. 2). Therefore we named the two subpopulations as hspLadakh and hspIndia respectively. For the 13 Malaysian isolates falling into hpEurope, three Indian and three Malay isolates belonged to AE1 while one Chinese, five Indian and one Malay isolate belonged to AE2.

\section{Identification of polymorphisms distinguishing the subpopulations}

Based on above STRUCTURE analysis, we reasoned that there must be informative bases that support the division of the subpopulations. To identify these bases, we performed site-by-site pairwise comparisons between subpopulations using Fisher's exact test at a significance level of 0.05 with Dunn-Sidak correction for multiple site comparisons. We examined five subpopulations in four comparisons, hspLadakh versus hspIndia, hspEAsia versus hspIndia, hspEAsia versus hspMaori, and hspEAsia versus hspAmerind subpopulations. Out of the 413, 377, 362 and 377 informative sites in the four pairwise comparisons, 27, 48, 39 and 32 sites respectively support the population divisions and we define these sites as population segregation sites (PSSs) (Table 1 and Fig. 3). The gene containing the most PSSs was $\operatorname{trp} C$ which was also the most variable gene while the gene carrying the fewest number of PSSs was ppa with zero or one site. The sites supporting one subpopulation division may not support another population division.

The patterns of the PSSs also provided further insight into recombination between populations. STRUCTURE analysis showed that in all subpopulations there were isolates with genes from other populations but the analysis did not identify which gene contributes to the mosaic genetic background. As shown in Figure 3 for hspIndia and hspLadakh comparison, the PSSs clearly showed the origin of some imported genes. Some involved the whole gene while others only involved segments of a gene. Many of these recombinational events must have occurred in the original population in India. The identification of the PSSs supports the results of STRUCTURE analysis which showed 8.9 to $33.2 \%$ imports and for the first time allowed us to identify the ancient alleles or sites in the populations concerned. The total number of PSSs between populations also reflects the distance between them. The more distantly related populations carry more segregating sites.

\section{Isolates with identical alleles}

H. pylori has been reported to be clonal only over a short period of time [11] and thus identical alleles among isolates is expected to be rare when sampling a large population. Interestingly, among the 78 Malaysian isolates analysed, 14 isolates had one or more identical alleles to other isolates. Two pairs of isolates, FD584i/FD589i, and FD419m/FD433m were identical in all seven genes; one pair of isolates, GC48i and FD566c, shared six identical genes; two pairs of isolates, FD539i and FD523i, and FD616i and FD540i share four identical genes; another two pairs of isolates, FD529c and FD519c, and FD556i and FD574i shared two identical genes and seven sets of isolates of 2-5 isolates shared one identical gene. Most of the identical genes were shared among the same ethnic 
A.

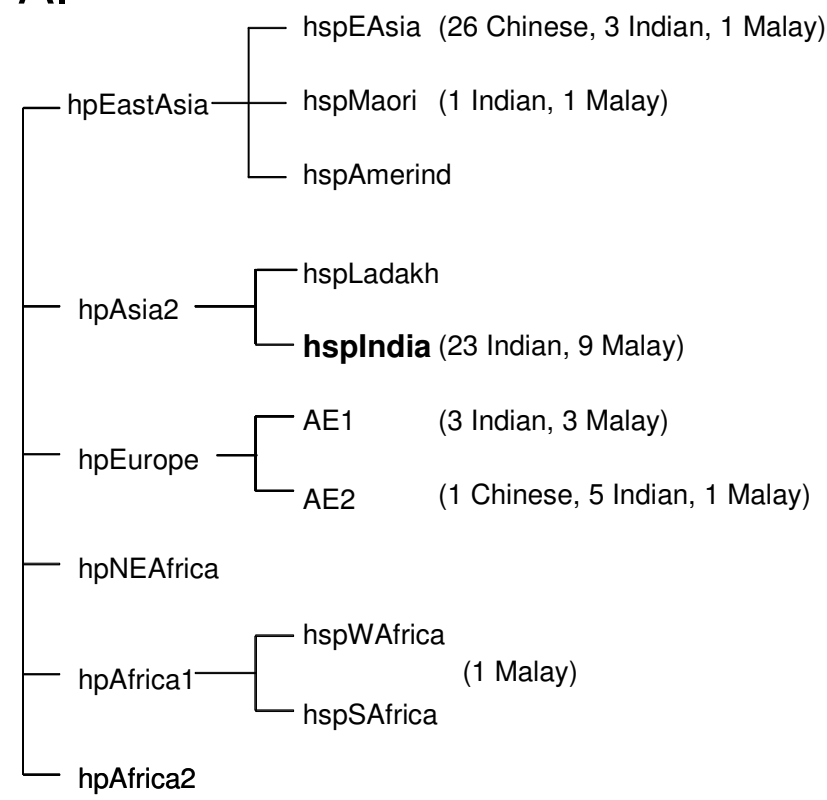

B.

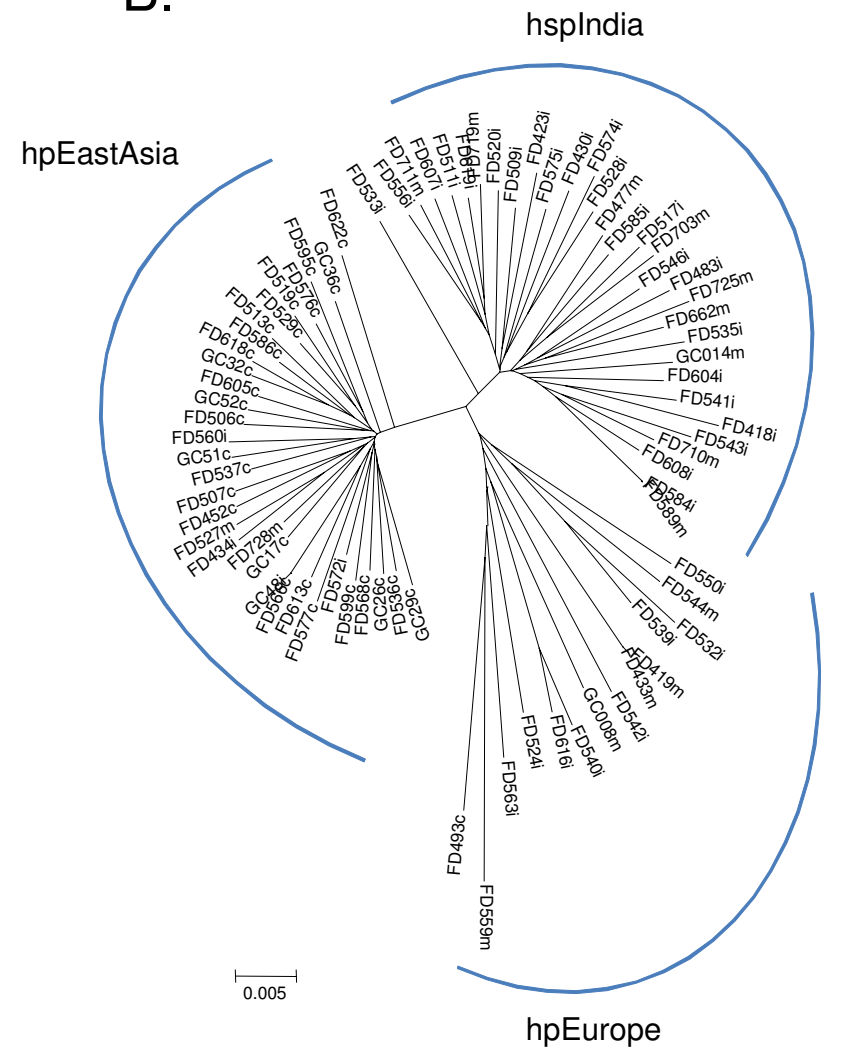

Figure I

Population and phylogenetic structure of the Malaysian isolates. A) Ancestral populations and population assignment of the Malaysian isolates. The division into populations and subpopulations according to Falush et al. [12] and Linz et al. [2] with the new subpopulation identified in this study in bold. The number of isolates from this study falling into each subpopulation or population is shown in brackets. B) Neighbour joining tree of the Malaysian isolates. 


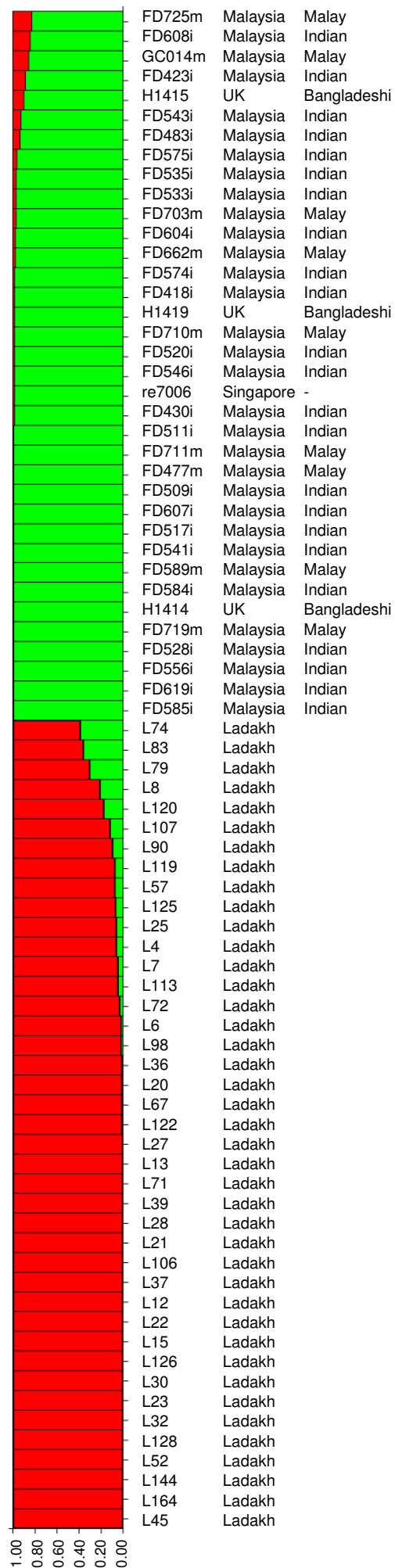

Figure 2

Division of hpAsia2 into subpopulations by STRUCTURE analysis. The two subpopulations, hspLadakh (red) and hsplndia (green) and assignment of isolates were shown. Each horizontal bar represents an isolate with isolate names and population and/or ethnic origin shown on the right. All Malaysian isolates were from this study while other isolates from the global MLST data. Mosaic colours for an isolate indicate mixed population origin from respective populations of matching colour. $Y$ axis represents percentage of population assignment. 


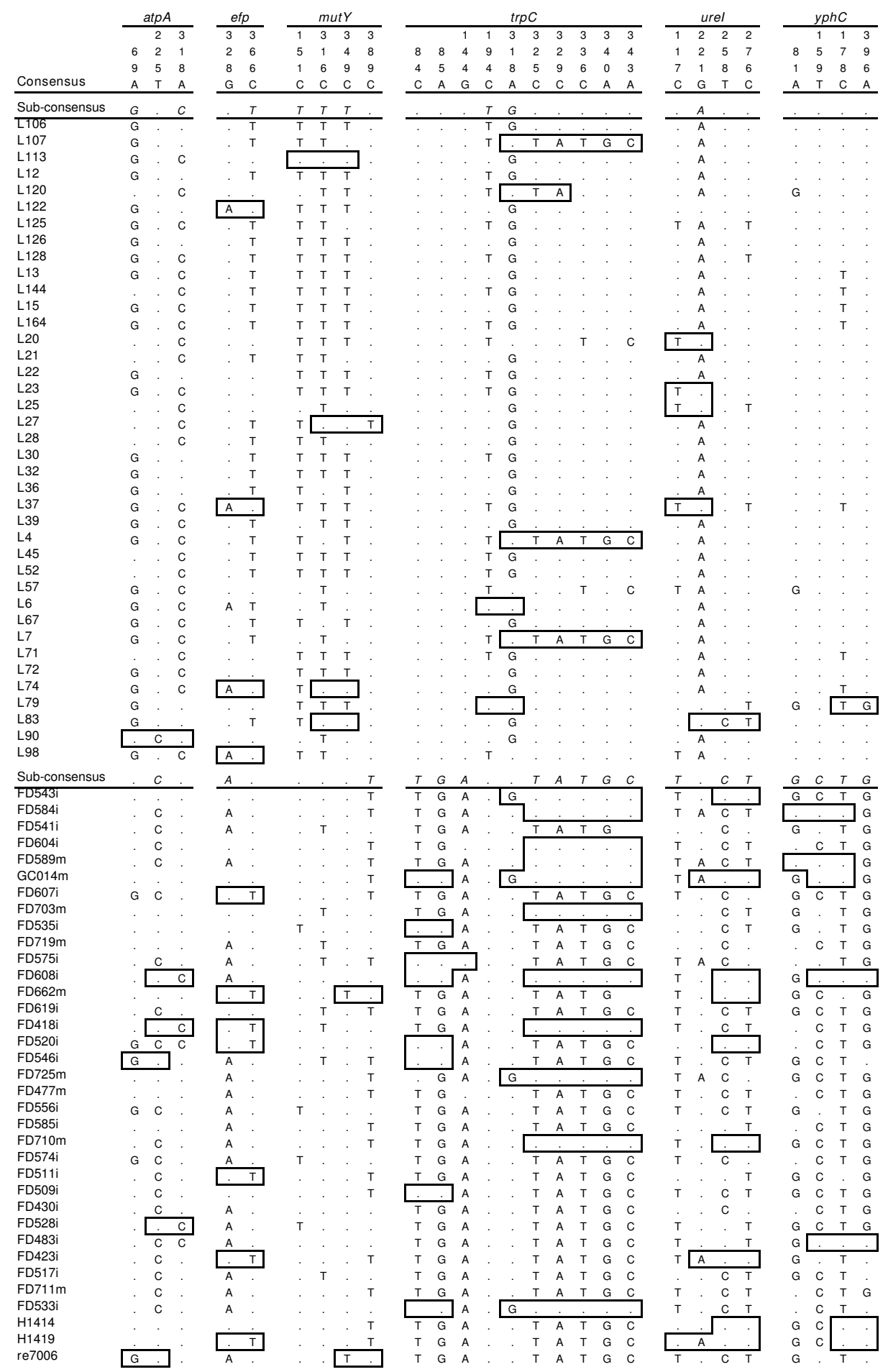

\section{Figure 3}

Population segregation sites between hsplndia and hspLadakh. The overall consensus is shown at the top. Subpopulation consensus is shown above each subpopulation. Boxed sites shown are segments with at least two identical population segregation sites to the other population. Ladakh isolates (with names starting $\mathrm{L}$ ) on the top panel and isolates from this study at the bottom panel. Genes and site positions were shown at top (reading vertically). ppa is not shown as it has no population segregation sites. 
population. However, we did observe that some genes were shared by different ethnic populations, most of which share only one identical gene. An Indian isolate (GC48i) shared six identical genes with a Chinese isolate (FD566c) and another Indian isolate (FD560i) had an identical gene with three Chinese isolates (FD586c, GC26c and GC52c).

We extended our analysis to include the 423 global isolate data to screen for identical genes that were shared globally. Fourteen pairs of isolates had all seven genes identical. There were $12,6,14,15,20,35$ sets with at least two isolates in each set sharing exclusively 6, 5, 4, 3, 2, and 1 identical alleles respectively. In a small number of cases a single isolate shared a subset of alleles with isolates that had a higher number of identical alleles these isolates were excluded. Isolates shared the most alleles in the efp gene and the least in ureI and $y p h C$.

\section{Discussion \\ Population Structure of H. pylori among Malaysian Populations}

H. pylori has been shown to have migrated with its host out of Africa $[2,12]$ and population differentiation is seen clearly in different regions of the world. However no studies have looked at recent $H$. pylori migration histories. Malaysia has a history of human immigration divided into three major waves, the earliest human settlement by the Orang Asli people - the Malay aborigines, the migration of current Malays 3000 years ago, and the mid-nineteenth century migration of Chinese and Indians. There is no data on $H$. pylori infection in the Orang Asli people, but good studies of the other three major ethnic populations are available $[22,23,26]$. The $H$. pylori infection rate and disease severity are different among the three ethnic populations. This population mixture in Malaysia provided a good opportunity to determine the $H$. pylori population admixture and to enhance our understanding of differences in infection rate and disease severity. We have shown in this study that the isolates recovered from the Malaysian H. pylori population belong to three of the known $H$. pylori ancestral populations, hpEastAsia, hpAsia 2 and hpEurope. The H. pylori isolates from the Chinese and Indian individuals were divided along their ethnic origins. Surprisingly the Malay isolates did not have a separate origin which is discussed below. There were six Indian isolates having Chinese $H$. pylori ancestry but none the reverse.

The population divisions identified in the current study are supported by the distribution of the cagA phosphorylation motif EPIYA [27] and vacA alleles [26] reported in these populations. The predominant EPIYA motif in the Malaysian Chinese population has been shown to be ABD $(87.8 \%)$ while the predominant type in both the Malay- sian Indian and the Malay populations is $\mathrm{ABC}$ with a frequency of $60.5 \%$ and $46.2 \%$ respectively. For vacA, the predominant genotype has been reported to be s1a among the Malaysian Malay (76.6\%) and Indian populations $(71.0 \%)$, and s1c among the Malaysian Chinese population $(66.1 \%)$ [26]. Data from these two genes confirm our observation that the Malay $H$. pylori population is more similar to Indian than to Chinese population.

It has been suggested that the combined effect of high levels of recombination and diversity does not allow phylogenetic analysis of $H$. pylori isolates $[2,12]$ and also implies that one would not expect to find any identical alleles to be recovered from the population unless they are from related hosts. However for the first time, we uncovered isolates with identical alleles, ranging from one to seven alleles, within and between the three Malaysian populations. The available patient medical information showed that these isolates were not from related hosts. We also found isolates with up to seven identical alleles present in the global MLST data, which was not described previously. The recovery of isolates with identical alleles indicates that the frequency of recombination may be lower and hence clones may be more stable than previously thought. We suggest that isolates with even one identical allele are likely to be related by decent rather than recent recombination involving that gene. Thus it may be possible to determine relationships of isolates if more genes are sequenced.

\section{The origin of Malay $\mathbf{H}$. pylori}

The Malay $H$. pylori population did not form a group of its own. The majority (nine of the 16 isolates studied) belong to the same group as the Indian isolates. Clearly the Malay isolates share the same origin as the Indian isolates. This conclusion has a number of implications for the origin of the Malay people and Malay H. pylori. Previous studies have shown that $H$. pylori follows the human route of migration and reflects human ancestry. However there is no evidence that ancestral Malays migrated from India. Currently there are two theories for the origins of Malay [28], one being of Southeast Asian origin, specifically sharing common ancestry with the Thais, the Laotians and the Cambodians while the other of Southern China origin through migration to Taiwan, then outwards to the Philippines, Borneo, Indonesia and Malaysia. The latter theory is supported by language origins while the former is supported by genetic evidence [28]. Neither supports Malays sharing direct common ancestry with Indians. Therefore for the Malay population, the ancestry of $H$. pylori does not reflect human ancestry as in other populations.

This raises the question as to what happened with the original Malay $H$. pylori since the human population 
undoubtedly carried the bacterium before migrating out of Africa. Studies showed that the H. pylori infection rate in the Malay population is much lower than that in the Indian population [22]. It is therefore likely that the Malay population was initially free of $H$. pylori and that the H. pylori in the current Malay population has only recently been acquired from the Malaysian Indian community. It is possible that the Malay population lost its original $H$. pylori [29]. However loss of $H$. pylori in modern populations is associated with improved living standards and this would be unlikely to be a plausible explanation for the initial loss of $H$. pylori in the Malay population.

While the Indian and Chinese populations have a small percentage of isolates from populations other than their ancestral populations (ie hspIndia and hspEAsia respectively), the Malay population has a much higher proportion of isolates (7 of the 16 isolates studied, 43.75\%) from populations other than hspIndia (see discussion below). This adds support to the hypothesis that the Malay population was initially free from $H$. pylori and that these isolates were directly imported from other populations recently. The higher proportion of Malay isolates from the Indian population than from the Chinese population suggests that there has been greater direct interaction between the Malay and Indian populations than between the Malay and Chinese populations. Reduced interaction between the Malays and Chinese may relate to factors such as incompatibility in food sources (eg pork) [30] which may have partly constrained the transmission of $H$. pylori from the Chinese to the Malay population.

Another potential source of $H$. pylori for non-aboriginal Malays is the Orang Asli population, who originated from early human migration out of Africa. The Orang Asli is likely to have taken the "Southern Route" into South East Asia to reach Malaysia by traveling along the Indian Ocean Coast line 50-65,000 years ago [31-33]. Therefore the Orang Asli $H$. pylori, if it exists, may share common ancestry with the Indian $H$. pylori, leading to the observed similarity of Malay isolates to Indian isolates. However given that other earlier $H$. pylori populations such as the Maori and American Indian populations can be readily identified [12], one would expect that the Orang Asli $H$. pylori population would be unique and identifiable after such a long period of separation, arguing against acquisition from Orang Asli population and in favour of acquisition from the Indian population.

\section{Flow of $\mathrm{H}$. pylori genes/genotypes among the Malaysian population and from other populations}

Apart from the Malay population who appear to have gained the majority of its $\mathrm{H}$. pylori isolates from the Indian population as discussed above, there was also gene flow from other populations. In particular the Indian and
Malay populations have higher levels of inflow of genes. Thirteen of the 51 (25.5\%) Malaysian Indian/Malay isolates were found grouped with the hpEurope population: six isolates grouped with AE1 and seven with AE2 (Additional file 1). One Malay isolate was found to be grouped with hpAfrica1, and one Indian and one Malay isolates grouped with hspMaori. The Malaysian Chinese population seems to have little inflow of genes from other populations with the exception of one Chinese isolate which grouped with AE2. The low frequency of Chinese isolates with other population affinity indicates that this isolate was more likely to have been acquired by its current or most recent host directly from an AE2 H. pylori host.

In contrast, the Indian/Malay isolates with ancestral European history (Table 2) are more likely to represent greater heterogeneity in the Indian/Malay $H$. pylori population and not direct transmission of isolates from the current European population or from early British or Portuguese colonization as these strains have genes from the Indian H. pylori gene pool. These isolates contain $8 \%$ to $40 \%$ hspIndia genes based on STRUCTURE analysis. By population segregation sites, 14 segments with at least two PSSs identical to the Indian/Malay population were identified (data not shown). Three isolates have one identical (PSSs) allele (FD542i in atpA, FD550i in mutY, FD540i in ureI). In contrast, the only Chinese isolate (FD493c) with a European ancestry showed almost no signal of Indian or Chinese ancestry. Such a diversity of isolates in the Malaysian population is interesting and warrants further studies.

There are two other possible sources of genetic heterogeneity in the Indian $H$. pylori: century-old importation and earlier common ancestry. While the colonisation of India from as early as the fifteenth century by the Portuguese and later by the British Empire [34] may have contributed directly to the Indian $H$. pylori gene pool from the European population. This explanation does not reconcile well with the observation by Wirth et al. [35] that in the Ladakh population genes of European ancestry were found despite the population being in a more pristine region. It has previously been suggested that AE1 originated in Central Asia because it shares phylogenetic signals with isolates from Estonia, Finland and Ladakh [12,36]. Since the ancestors of Malaysian Indians and the Ladakhis resided in the same region and their $H$. pylori belong to hpAsia2, it seems likely that this biased share of the gene pool by the Malaysian Indian/Malay isolates with the AE1 subpopulation is due to an earlier common ancestry. Our findings are consistent with the studies of Wirth et al. [35], Linz et al. [2] and Devi et al. [19] that H. pylori in the Indian population is more heterogeneous in origin, reflecting perhaps both earlier common ancestry and recent imports. 
Table 2: List of strains and population affinity based on STRUCTURE analysis

\begin{tabular}{|c|c|c|c|c|c|}
\hline Strain ID & Disease & Ethnic origin & Population affinity $(\%)^{\$}$ & Population & subpopulation \\
\hline FD4I8i & Functional dyspepsia & Indian & 81 & hpAsia2 & hsplndia \\
\hline FD423i & Functional dyspepsia & Indian & 72 & hpAsia2 & hsplndia \\
\hline FD430i & Functional dyspepsia & Indian & 78 & hpAsia2 & hsplndia \\
\hline FD434i & Functional dyspepsia & Indian & 96 & hpEastAsia & hspMaori \\
\hline FD483i & Functional dyspepsia & Indian & 84 & hpAsia2 & hsplndia \\
\hline FD509i & Functional dyspepsia & Indian & 77 & hpAsia2 & hsplndia \\
\hline FD5IIi & Functional dyspepsia & Indian & 75 & hpAsia2 & hsplndia \\
\hline FD5I7i & Functional dyspepsia & Indian & 78 & hpAsia2 & hsplndia \\
\hline FD520i & Functional dyspepsia & Indian & 83 & hpAsia2 & hsplndia \\
\hline FD524i & Functional dyspepsia & Indian & 54 & hpEurope & AE2 \\
\hline FD528i & Functional dyspepsia & Indian & 77 & hpAsia2 & hsplndia \\
\hline FD532i & Functional dyspepsia & Indian & 46 & hpEurope & $A E I$ \\
\hline FD533i & Functional dyspepsia & Indian & 71 & hpAsia2 & hsplndia \\
\hline FD535i & Functional dyspepsia & Indian & 88 & hpAsia2 & hsplndia \\
\hline FD539i & Functional dyspepsia & Indian & 62 & hpEurope & $\mathrm{AEI}$ \\
\hline FD540i & Functional dyspepsia & Indian & 67 & hpEurope & AE2 \\
\hline FD54li & Functional dyspepsia & Indian & 81 & hpAsia2 & hsplndia \\
\hline FD542i & Functional dyspepsia & Indian & 40 & hpEurope & $\mathrm{AEI}$ \\
\hline FD543i & Functional dyspepsia & Indian & 86 & hpAsia2 & hsplndia \\
\hline FD546i & Functional dyspepsia & Indian & 81 & hpAsia2 & hsplndia \\
\hline FD550i & Functional dyspepsia & Indian & 48 & hpEurope & AE2 \\
\hline FD556i & Functional dyspepsia & Indian & 82 & hpAsia2 & hsplndia \\
\hline FD560i & Functional dyspepsia & Indian & 90 & hpEastAsia & hspEAsia \\
\hline FD563i & Functional dyspepsia & Indian & 54 & hpEurope & AE2 \\
\hline FD572i & Functional dyspepsia & Indian & 97 & hpEastAsia & hspEAsia \\
\hline FD574i & Functional dyspepsia & Indian & 82 & hpAsia2 & hsplndia \\
\hline FD575i & Functional dyspepsia & Indian & 86 & hpAsia2 & hsplndia \\
\hline FD584i & Functional dyspepsia & Indian & 85 & hpAsia2 & hsplndia \\
\hline FD585i & Functional dyspepsia & Indian & 71 & hpAsia2 & hsplndia \\
\hline FD604i & Functional dyspepsia & Indian & 88 & hpAsia2 & hsplndia \\
\hline FD607i & Functional dyspepsia & Indian & 84 & hpAsia2 & hsplndia \\
\hline FD608i & Functional dyspepsia & Indian & 83 & hpAsia2 & hsplndia \\
\hline FD616i & Functional dyspepsia & Indian & 50 & hpEurope & AE2 \\
\hline FD619i & Functional dyspepsia & Indian & 79 & hpAsia2 & hsplndia \\
\hline GC48i & Functional dyspepsia & Indian & 95 & hpEastAsia & hspEAsia \\
\hline FD452c & Functional dyspepsia & Chinese & 91 & hpEastAsia & hspEAsia \\
\hline FD493c & Functional dyspepsia & Chinese & 63 & hpEurope & AE2 \\
\hline FD506c & Functional dyspepsia & Chinese & 97 & hpEastAsia & hspEAsia \\
\hline FD507c & Functional dyspepsia & Chinese & 96 & hpEastAsia & hspEAsia \\
\hline FD5I3c & Functional dyspepsia & Chinese & 86 & hpEastAsia & hspEAsia \\
\hline FD519c & Functional dyspepsia & Chinese & 89 & hpEastAsia & hspEAsia \\
\hline FD529c & Functional dyspepsia & Chinese & 97 & hpEastAsia & hspEAsia \\
\hline FD536c & Functional dyspepsia & Chinese & 95 & hpEastAsia & hspEAsia \\
\hline FD537c & Functional dyspepsia & Chinese & 92 & hpEastAsia & hspEAsia \\
\hline FD566c & Functional dyspepsia & Chinese & 95 & hpEastAsia & hspEAsia \\
\hline FD568c & Functional dyspepsia & Chinese & 93 & hpEastAsia & hspEAsia \\
\hline FD576c & Functional dyspepsia & Chinese & 98 & hpEastAsia & hspEAsia \\
\hline FD577c & Functional dyspepsia & Chinese & 85 & hpEastAsia & hspEAsia \\
\hline FD586c & Functional dyspepsia & Chinese & 93 & hpEastAsia & hspEAsia \\
\hline FD595c & Functional dyspepsia & Chinese & 83 & hpEastAsia & hspEAsia \\
\hline FD599c & Functional dyspepsia & Chinese & 94 & hpEastAsia & hspEAsia \\
\hline FD $605 c$ & Functional dyspepsia & Chinese & 93 & hpEastAsia & hspEAsia \\
\hline FD613c & Functional dyspepsia & Chinese & 95 & hpEastAsia & hspEAsia \\
\hline FD618c & Functional dyspepsia & Chinese & 90 & hpEastAsia & hspEAsia \\
\hline FD622c & Functional dyspepsia & Chinese & 83 & hpEastAsia & hspEAsia \\
\hline $\mathrm{GCI}$ c & Gastric Cancer & Chinese & 89 & hpEastAsia & hspEAsia \\
\hline GC26c & Gastric Cancer & Chinese & 92 & hpEastAsia & hspEAsia \\
\hline GC29c & Functional dyspepsia & Chinese & 95 & hpEastAsia & hspEAsia \\
\hline GC32c & Gastric Cancer & Chinese & 89 & hpEastAsia & hspEAsia \\
\hline
\end{tabular}


Table 2: List of strains and population affinity based on STRUCTURE analysis (Continued)

\begin{tabular}{|c|c|c|c|c|c|}
\hline GC36c & Gastric Cancer & Chinese & 88 & hpEastAsia & hspEAsia \\
\hline GC5IC & Gastric Cancer & Chinese & 94 & hpEastAsia & hspEAsia \\
\hline GC52c & Gastric Cancer & Chinese & 95 & hpEastAsia & hspEAsia \\
\hline FD419m & Functional dyspepsia & Malay & 56 & hpEurope & $\mathrm{AEI}$ \\
\hline FD433m & Functional dyspepsia & Malay & 57 & hpEurope & AEI \\
\hline FD477m & Functional dyspepsia & Malay & 73 & hpAsia2 & hsplndia \\
\hline FD527m & Functional dyspepsia & Malay & 88 & hpEastAsia & hspMaori \\
\hline FD544m & Functional dyspepsia & Malay & 59 & hpEurope & $\mathrm{AEI}$ \\
\hline FD559m & Functional dyspepsia & Malay & 69 & hpAfrical & unknown \\
\hline FD589m & Functional dyspepsia & Malay & 86 & hpAsia2 & hsplndia \\
\hline FD $662 \mathrm{~m}$ & Functional dyspepsia & Malay & 86 & hpAsia2 & hsplndia \\
\hline FD703m & Functional dyspepsia & Malay & 83 & hpAsia2 & hsplndia \\
\hline FD7IOm & Functional dyspepsia & Malay & 83 & hpAsia2 & hsplndia \\
\hline FD7I Im & Functional dyspepsia & Malay & 73 & hpAsia2 & hsplndia \\
\hline FD7I9m & Functional dyspepsia & Malay & 75 & hpAsia2 & hsplndia \\
\hline FD725m & Functional dyspepsia & Malay & 82 & hpAsia2 & hsplndia \\
\hline FD728m & Functional dyspepsia & Malay & 97 & hpEastAsia & hspEAsia \\
\hline GC008m & Functional dyspepsia & Malay & 53 & hpEurope & AE2 \\
\hline $\mathrm{GCOI} 4 \mathrm{~m}$ & Gastric Cancer & Malay & 89 & hpAsia2 & hsplndia \\
\hline
\end{tabular}

\$ Denotes \% genes allocated to the given population by STRUCTURE analysis

\section{Division of hpAsia2 into subpopulations}

The hpAsia2 population was initially defined based on isolates from Ladakh in Northern India, which represents the west/middle Asia population. Since the Malaysian Indian population is known to have originated from India, the Malaysian Indian isolates were initially not expected to be distinguishable from the Ladakh population. However, we have shown that the two populations can be divided within hpAsia2 as subpopulations, hspLadakh and hspIndia (Fig. 2). A total of 27 (or 0.91\%) segregating sites among the seven housekeeping genes were identified to separate the two subpopulations. There is however considerable gene flow between the two populations. Identical alleles as defined by the PSSs can be treated as recombination that occurred in the more distant past. These alleles are present in three genes (atpA, efp and ureI). Further many segments with at least two identical PSSs are present in three other genes (mutY, $\operatorname{trpC}$ and $y p h C$; Fig. 3). Note that $p p a$ has no PSSs. These results suggest that there is considerable population admixture in the earlier history of the Indian population. A recent study of the Indian population sequenced 23 isolates by MLST but the sequences are shorter [19]. STRUCTURE analysis of combined data from our Malaysian Indian isolates, Ladakh isolates and these 23 Indian isolates using $\mathrm{k}=2$ populations and found that the Malaysian Indian isolates grouped together with the Indian isolates while the Ladakh isolates were separate. However, when $k=3$ populations were used, the two sets of Indian isolates were separated (data not shown). This suggests that the two Indian populations overlap but are distinctive. The Malaysian Indian $H$. pylori population may have differentiated further from the Indian H. pylori population from India, although it is also possible that the difference between the two $H$. pylori populations reflects regional differences in
India as the Malaysian Indians mainly came from South India.

\section{Conclusion}

This study has shown that the Malaysian H. pylori isolates can be differentiated into three populations using MLST, being hpEastAsia, hpAsia2 and hpEurope. Interestingly the Malay population was shown to carry $H$. pylori isolates of Indian origin. The infection rate of $H$. pylori among the Malay population is low in comparison to the Malaysian Indian population [22]. In western countries a low or reduced rate of $H$. pylori infection is attributed to high or improved hygiene standard [3]. However this factor does not account for differences between the Malay and the other two populations $[21,22]$. Therefore the Malay population was likely to be initially $H$. pylori-free and has acquired $H$. pylori only recently from the Indian population. Thus the low H. pylori infection rate in the Malay population may be due to low cross infection rate from another population.

The Malaysian Indian/Malay isolates were found to differ from the Ladakh isolates from India and in fact formed a new subpopulation, hspIndia. Clearly there are more subpopulations of $H$. pylori and populations can be divided at a finer scale when more isolates are used or more geographical regions are sampled. More extensive worldwide surveys will help us further understand the evolution and population structure of $H$. pylori, an organism that has impacted more than half of the world's population and continues to pose great risk to human health because of its association with gastric cancer and MALT lymphoma. Genetic heterogeneity of the bacterium within a host population as shown in this study should be taken into account when studying the epidemiology and pathogene- 
sis of $H$. pylori since there is clearly variation in incidence and severity of the disease in different populations.

\section{Methods}

Source of gastric biopsies and culture of $\mathbf{H}$. pylori isolates Gastric biopsies were collected as part of a large-scale gastric cancer study conducted in symptomatic patients undergoing gastroenterological examination at the Faculty of Medicine, University of Malaya, Kuala Lumpur, Malaysia. All biopsies were obtained with the informed consent of the patients and this study was approved by the Human Ethics Committees of the University of New South Wales and the University of Malaya. Based on endoscopic and histological examinations, patients were diagnosed as having gastric cancer or functional dyspepsia. All except seven samples were from patients with functional dyspepsia as shown in Table 2.

H. pylori was cultured by inoculating biopsies on Campylobacter selective agar (CSA) containing 4\% blood base agar No. 2 (Oxoid), defibrinated horse blood (Oxoid), and one vial of Skirrow's supplement (Oxoid) containing 2.5 mg Trimethoprim, $5.0 \mathrm{mg}$ Vancomycin, and 1250 IU polymyxin B. Primary cultures were incubated at $37^{\circ} \mathrm{C}$ with $10 \% \mathrm{CO}_{2}$ in a $\mathrm{CO}_{2}$ incubator (Plymouth, USA) for up to 10 days, observing daily for growth. For isolation of pure cultures a single colony was picked and subcultured onto CSA for four days. Identification of $H$. pylori was based on microscopic morphology and biochemical testing (urease, oxidase and catalase). One isolate from each biopsy was selected for this study and 78 isolates were obtained from patients of different ethnic background, including 27 Chinese, 35 Indian and 16 Malay (Table 2). We used all Malay biopsy samples available. Despite the fact that this study spanned a period of four years the number of Malay subjects from whom $H$. pylori could be cultured was low which reflects the relative low prevalence in this population. Isolates from this study are available to researchers upon request to HM.

\section{Chromosomal DNA purification}

One plateful of bacterial culture was collected and suspended into $215 \mu \mathrm{l}$ of Tris $(50 \mathrm{mM}), 15 \mu \mathrm{l}$ of EDTA $(0.5$ $\mathrm{M})$ and incubated for $10 \mathrm{~min}$. Two $\mu \mathrm{l}$ of proteinase $\mathrm{K}(10$ $\mathrm{mg} / \mathrm{ml}$ ) and $20 \mu \mathrm{l}$ of SDS (10\%) were added followed by incubation at $50^{\circ} \mathrm{C}$ for a minimum of $2 \mathrm{~h}$ or until clear. One $\mu$ l of RNase $(10 \mathrm{mg} / \mathrm{ml})$ was added and incubated at $65^{\circ} \mathrm{C}$ for an additional $20 \mathrm{~min}$. the mixture was then transferred into a $1.5 \mathrm{ml}$ Heavy Gel Phase tube (Eppendorf) and washed twice with one volume of phenol:chloroform:isoamyl alcohol (25:24:1) and once with one volume of chloroform:isoamyl alcohol (24:1). The top layer was transferred into a new $1.5 \mathrm{ml}$ tube containing $600 \mu \mathrm{l}$ of pre-chilled EtOH (100\%). Precipitated DNA was then spooled out, washed in $70 \%(\mathrm{v} / \mathrm{v}) \mathrm{EtOH}$, dissolved in $100 \mu \mathrm{l}$ TE buffer ( $10 \mathrm{mM}$ Tris $1 \mathrm{mM}$ EDTA, pH 8.0) and incubated at $65^{\circ} \mathrm{C}$ for $15 \mathrm{~min}$ to evaporate the residual ethanol.

\section{PCR assay and DNA sequencing}

The primer sequences for MLST of the seven house keeping genes used in this study were those described by Achtman et al. [10]. Primers were synthesized commercially (Sigma-Aldrich). Each PCR reaction included 2.0 $\mu$ l DNA template (approx. $20 \mathrm{ng}), 0.5 \mu \mathrm{l}(30 \mathrm{pmol} / \mu \mathrm{l})$ of each forward and reverse primer, $0.5 \mu \mathrm{l}$ of $\mathrm{dNTP}(10 \mathrm{mM}), 5 \mu \mathrm{l}$ of $10 \times$ PCR buffer $(500 \mathrm{mM} \mathrm{KCl}, 100 \mathrm{mM}$ Tris-HCl, $\mathrm{pH} 9.0$, $1 \%$ Triton $\mathrm{X}-100$ and $15 \mathrm{mM} \mathrm{MgCl}_{2}$ ), $0.25 \mu \mathrm{l}$ of Taq polymerase (1.25 U) and MilliQ water to a total volume of $50 \mu \mathrm{l}$. PCR cycles were performed in a Hybaid PCR Sprint Thermocycler (Hybaid): initial DNA denaturation for $2 \mathrm{~min}$ at $94^{\circ} \mathrm{C}$, followed by DNA denaturation for 15 sec at $94^{\circ} \mathrm{C}$, primer annealing for $30 \mathrm{sec}$ at $50^{\circ} \mathrm{C}$, and polymerization for $90 \mathrm{sec}$ at $72^{\circ} \mathrm{C}$ for 35 cycles, with a final extension of $5 \mathrm{~min}$ at $72^{\circ} \mathrm{C}$. PCR products were verified on ethidium bromide stained agarose gels. PCR product for sequencing was purified using sodium acetate/ethanol precipitation. The 20- $\mu$ l PCR sequencing mixture contained $1 \mu \mathrm{l}$ of BigDye (version 3.1; Applied Biosystems), $20 \mathrm{ng}$ of the purified PCR product, $3.5 \mu \mathrm{l}$ of $5 \times$ PCR sequencing buffer (Applied Biosystems), $1 \mu \mathrm{l}$ of forward primer (concentration, $3.2 \mathrm{pmol} / \mu \mathrm{l}$; SigmaAldrich), and MilliQ water. Unincorporated dye was removed by ethanol precipitation. The sequencing reaction mixtures were resolved on an ABI 3730 automated DNA sequence analyzer (Applied Biosystems) at the sequencing facility of the School of Biotechnology and Biomolecular Sciences, University of New South Wales, Sydney, Australia.

\section{Bioinformatic analysis}

PHRED PHRAP and CONSED [37] program package, accessed through the Australia National Genomic Information Service, was used for sequence editing. PILEUP from the Genetics Computer Group package [38], and MULTICOMP [39], were used for multiple sequence alignment and comparison. PHYLIP [40] was used to generate phylogenetic trees. STRUCTURE version 2.2 [25], which implements a Bayesian approach for deducing population structure from multilocus data, was used to analyse the population clustering of an isolate, assuming that each isolate has derived all of its ancestry from only one population. The number of populations, $K$, was determined under the "no admixture" model and in each simulation run, the Markov Chain Monte Carlo (MCMC) simulation of 30,000 iterations approximated the posterior probability of $K$, following a burn-in of 10,000 iterations. After multiple runs on each $K$ assumed, the value that generated the highest posterior probability was used as the number of possible populations. The assignment of an isolate to a particular population was done under the linkage model. 


\section{Segregation site Analysis}

Gene sequences from two populations were aligned and compiled by PILEUP and MULTICOMP. Informative sites, which are defined as those with at least two variants at a particular site and more than one isolate for each base variant, were extracted from output generated by MULTICOMP and examined using Microsoft EXCEL. Total base changes at each informative site present in each population were summed and formed a $2 \times 2$ table for Fisher's Exact test using SPSS (SPSS Inc, Chicago, IL). For those informative sites that have more than two variants, the least frequent base was removed and treated as a missing value. The probability of each site generated by SPSS was adjusted using Dunn-Sidak correction: $\alpha^{\prime}=1-(1-\alpha)^{1 / p}$, where $\alpha^{\prime}$ represent adjusted probability, $\alpha$ represent the significance value ( 0.05 used in this study) and p represent the total number of comparisons.

The GenBank accession numbers for the sequences reported in this study are FJ846683 - FJ847228.

\section{Authors' contributions}

RL conceived the study. CYT performed acquisition and analysis of data. HM and IWD participated in its design and coordination. QD participated in data acquisition. KLG contributed to the materials. All authors participated in drafting the manuscript, and read and approved the final manuscript.

\section{Additional material}

\section{Additional file 1}

STRUCTURE analysis of Malaysian and global isolates. The data provided represent the population structure of global isolates and the distribution of Malaysian isolates.

Click here for file

[http://www.biomedcentral.com/content/supplementary/14712180-9-126-S1.pdf]

\section{Acknowledgements}

This study was supported by a University of New South Wales Goldstar award and the Cancer Council of New South Wales. We thank Heather Schmidt for providing some of the DNA samples and we thank the referees for helpful suggestions.

\section{References}

I. Covacci A, Telford JL, Giudice GD, Parsonnet J, Rappuoli R: Helicobacter pylori virulence and genetic geography. Science 1999, 284: |328-1333.

2. Linz B, Balloux F, Moodley Y, Manica A, Liu H, Roumagnac P, Falush D, Stamer C, Prugnolle F, Merwe SW van der, Yamaoka Y, Graham DY, Perez-Trallero E, Wadstrom T, Suerbaum S, Achtman M: An African origin for the intimate association between humans and Helicobacter pylori. Nature 2007, 445:915-918.

3. Mitchell HM: The epidemiology of Helicobacter pylori. In Gastroduodenal disease and Helicobacter pylori: Pathophysiology, Diagnosis and Treatment Edited by: Nedrud JG, Westblom U, Czinn S. Heidelberg: Springer Verlag; I998: I I-30.

4. Kuipers EJ, Israel DA, Kusters JG, Gerrits MM, Weel J, Ende A van der, Hulst RWM van der, Wirth HP, Höök-Nikanne JH, Thompson SA, et al:: Quasispecies development of Helicobacter pylori observed in paired Isolates obtained years apart from the same host. J Infect Dis 2000, I 8 I:273-282.

5. Pounder RR: The prevalence of Helicobacter pylori in different countries. Aliment Pharmacol Ther 1995, 9:33-40.

6. Parsonnet JE: The incidence of Helicobacter pylori infection. Aliment Pharmacol Ther 1995, 9:45-52.

7. Garner JA, TL C: Analysis of genetic diversity in cytotoxin-producing and non-cytotoxin-producing Helicobacter pylori strains. J Infect Dis 1995, I 72:290-293.

8. Ende A van der, Pan Z-J, Bart A, Hulst RWM van der, Feller M, Xiao S-D, Tytgat GNJ, Dankert J: cagA-Positive Helicobacter pylori Populations in China and The Netherlands Are Distinct. Infect Immun 1998, 66: I 822-1826.

9. Kansau I, Raymond J, Bingen E, Courcoux P, Kalach N, Bergeret M, Braimi N, Dupont C, A L: Genotyping of Helicobacter pylori isolates by sequencing of PCR products and comparison with the RAPD technique. Res Microbiol 1996, I47:66I-669.

10. Achtman M, Azuma T, Berg DE, Ito Y, Morelli G, Pan Z-J, Suerbaum S, Thompson SA, Ende A van der, van Doorn L-J: Recombination and clonal groupings within Helicobacter pylori from different geographical regions. Mol Microbiol 1999, 32:459-470.

II. Falush D, Kraft C, Taylor NS, Correa P, Fox JG, Achtman M, Suerbaum S: Recombination and mutation during long-term gastric colonization by Helicobacter pylori: estimates of clock rates, recombination size, and minimal age. Proc Natl Acad Sci USA 200I, 98:I5056-I506I.

12. Falush D, Wirth T, Linz B, Pritchard JK, Stephens M, Kidd M, Blaser MJ, Graham DY, Vacher S, Perez-Perez Gl, et al:: Traces of human migrations in Helicobacter pylori populations. Science 2003, 299: I582-1585

13. Lawrence JG, H O: Amelioration of bacterial genomes: rates of change and exchange. J Mol Evol 1997, 44:383-397.

14. Vlieland CA: The Population of the Malay Peninsula: A Study in Human Migration. Geogr Rev 1934, 24:61-78.

15. Andaya LY: The search for the 'origins' of Melayu. J Southeast Asian Stud 2001, 32:315-330.

16. Hirschman C: The meaning and measurement of Ethnicity in Malaysia: an analysis of census classifications. J Asian Stud I987, 46:555-582.

17. Hill C, Soares P, Mormina M, Macaulay V, Meehan W, Blackburn I, Clarke D, Raja JM, Ismail P, Bulbeck D, Oppenheimer S, Richards M: Phylogeography and ethnogenesis of aboriginal Southeast Asians. Mol Biol Evol 2006, 23:2480-249l.

18. Blaser MJ: Science, medicine, and the future - Helicobacter pylori and gastric diseases. $\mathrm{Br}$ Med J 1998, 3 I 6: I 507-I 510.

19. Devi SM, Ahmed I, Francalacci P, Hussain MA, Akhter Y, Alvi A, Sechi LA, Megraud F, Ahmed N: Ancestral European roots of Helicobacter pylori in India. BMC Genomics 2007, 8: 184

20. Parsonnet J: Helicobacter pylori - the size of the problem. Gut 1998, 43:S6-S9.

21. Goh KL, Parasakthi N: The racial cohort phenomenon: seroepidemiology of Helicobacter pylori infection in a multiracial South-East Asian country. Eur J Gastroenterol Hepatol 2001, 13:177-183.

22. Kaur G, Naing NN: Prevalence and ethnic distribution of Helicobacter pylori infection among endoscoped patients in north eastern peninsular Malaysia. Malaysian J Med Sci 2003, 10:66-70.

23. Boey CC, Goh KL, Lee WS, Parasakthi N: Seroprevalence of Helicobacter pylori infection in Malaysian children: evidence for ethnic differences in childhood. J Paediatr Child Health 1999 , 35: $|5|-152$

24. Goh KL, Cheah PL, Md N, Quek KF, Parasakthi N: Ethnicity and Helicobacter pylori as risk factors for gastric cancer in Malaysia: A prospective case control study. Am J Gastroenterol 2007, I 02:40-45.

25. Pritchard JK, Stephens M, Donnelly P: Inference of population structure using multilocus genotype data. Genetics 2000, I 55:945-959.

26. Tan HJ, Rizal AM, Rosmadi MY, Goh KL: Distribution of Helicobacter pylori cagA, cagE and vacA in different ethnic groups in 
Kuala Lumpur, Malaysia. J Gastroenterol Hepatol 2005, 20:589-594.

27. Schmidt H-MA, Goh KL, Fock KM, Hilmi I, Dhamodaran S, Forman D, Mitchell H: Distinct cagA EPIYA motifs are associated with ethnic diversity in Malaysia and Singapore. Helicobacter 2009 in press.

28. Ainoon O, Yu YH, Amir Muhriz AL, Boo NY, Cheong SK, Hamidah $\mathrm{NH}$ : Glucose-6-phosphate dehydrogenase (G6PD) variants in Malaysian Malays. Hum Mutat 2003, 2 1:101.

29. Graham DY, Yamaoka Y, Malaty HM: Thoughts about populations with unexpected low prevalences of Helicobacter pylori infection. Trans R Soc Trop Med Hyg 2007, I $01: 849-85$ I.

30. Kiong TC: The Chinese in contemporary Malaysia. In Race, Ethnicity, and the State in Malaysia and singapore Edited by: Fee LK. Leiden: Koninlijke Brill NV; 1996:95-II9.

31. Atkinson QD, Gray RD, Drummond AJ: mtDNA variation predicts population size in humans and reveals a major southern Asian chapter in human prehistory. Mol Biol Evol 2008, 25:468-474.

32. Forster P, Matsumura S: Did Early Humans Go North or South? Science 2005, 308:965-966.

33. Macaulay V, Hill C, Achilli A, Rengo C, Clarke D, Meehan W, Blackburn J, Semino O, Scozzari R, Cruciani F, Taha A, Shaari NK, Raja JM. Ismail P, Zainuddin Z, Goodwin W, Bulbeck D, Bandelt H-J, Oppenheimer S, Torroni A, Richards M: Single, Rapid Coastal Settlement of Asia Revealed by Analysis of Complete Mitochondrial Genomes. Science 2005, 308: 1034-1036.

34. Wolpert S: A New History of India. 7th edition. New York: Oxford University Press; 2003.

35. Wirth T, Wang X, Linz B, Novick RP, Lum JK, Blaser M, Morelli G, Falush D, Achtman M, Salzano FM: Distinguishing Human Ethnic Groups by Means of Sequences from Helicobacter pylori: Lessons from Ladakh. Proc Natl Acad Sci USA 2004, 10 I:4746-475 I.

36. Suerbaum S, Achtman M: Helicobacter pylori: recombination, population structure and human migrations. Int J Med Microbiol 2004, 294:133-139.

37. Gordon D, Abajian C, Green P: CONSED - A graphical tool for sequence finishing. Genome Res 1998, 8:195-202.

38. Dolz R: GCG. In Computer Analysis Of Sequence Data, Methods In Molecular Biology Edited by: Griffin AM, Griffin HG. Totpwa, NJ: Humana; 1994:9-17.

39. Reeves PR, Farnell L, Lan R: MULTICOMP: a program for preparing sequence data for phylogenetic analysis. Bioinformatics 1994, 10:281-284.

40. Felsenstein J: PHYLIP-phylogeny inference package. Cladistics 1989, 5:164-166.
Publish with Bio Med Central and every scientist can read your work free of charge

"BioMed Central will be the most significant development for disseminating the results of biomedical research in our lifetime. "

Sir Paul Nurse, Cancer Research UK

Your research papers will be:

- available free of charge to the entire biomedical community

- peer reviewed and published immediately upon acceptance

- cited in PubMed and archived on PubMed Central

- yours - you keep the copyright
BioMedcentral 